\title{
Kernos
}

Revue internationale et pluridisciplinaire de religion grecque antique

9 | 1996

Varia

\section{Fustel de Coulanges et La cité antique}

Pierre Somville

Édition électronique
URL : http://journals.openedition.org/kernos/1168

DOI : 10.4000/kernos. 1168

ISSN : 2034-7871

Éditeur

Centre international d'étude de la religion grecque antique

Édition imprimée

Date de publication : 1 janvier 1996

ISSN : 0776-3824

\section{Référence électronique}

Pierre Somville, «Fustel de Coulanges et La cité antique », Kernos [En ligne], 9 | 1996, mis en ligne le 21 avril 2011, consulté le 19 avril 2019. URL : http://journals.openedition.org/kernos/1168 ; DOI : 10.4000/kernos. 1768 


\section{Fustell de Coulanges et La Cité antique}

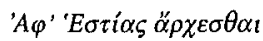

(Proverbe)

L'ouvrage écrit en 1864 reste d'une parfaite actualité. Les découvertes

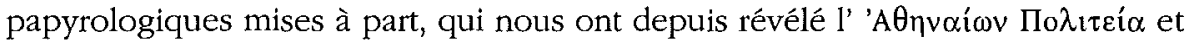
le Dyscolos de Ménandre, je ne vois pas ce qui lui manque. Couvrant les domaines de l'histoire grecque et romaine, il vaut à lui seul quatre cours d'« institutions », le caractère inséparable des aspects politiques et religieux desdites institutions apparaissant comme l'une des thèses principales de l'ouvrage. Bref, on ne saurait assez plaider pour sa redécouverte, sa relecture, sa réédition, son étude.

La méthode exemplaire - et quasi proverbiale - en est le retour aux sources, ou plutôt, la remontée aux sources, qu'il faut entendre de deux façons. D'abord, il s'agit de partir des textes antiques, rien que d'eux et d'en tirer tout ce qu'ils peuvent nous livrer, sans rien y ajouter. Ensuite, comme l'auteur l'affirme dès les premières lignes de son introduction, nous devons concevoir les sociétés antiques dans leur irréductible originalité. Les manifestations en sont spécifiques et nous ne devons pas plus nous y projeter que croire en tirer quelque leçon pour notre présent.

Cette double exigence d'objectivité, textuelle et anthropologique, fait de l'œuvre de Fustel de Coulanges ce véritable modèle de critique historique que nous disait à si juste titre notre maître Halkin.

Une des idées-clé de la Cité antique est l'importance de ce phénomène d'opinion, primordial et fondateur, qu'est la croyance religieuse. Loin de dériver d'un impératif politique ou d'un quelconque substrat économique, c'est d'elle plutôt que procèdent toutes les autres formes de l'activité sociale. Aux débuts de la Grèce et de Rome, à tout le moins. Le culte familial, sous la double espèce du feu domestique et du souvenir des ancêtres, dûment sacralisés, apparaît comme le premier point d'ancrage de ces sociétés indo-européennes qui constituèrent les cultures grecque et romaine.

Le chef de famille y est à la fois prêtre, roi et magistrat. C'est de lui que dépendent le culte, l'autorité et le droit. Lorsque l'état archaique se sera constitué, sur ce modèle privé, tout exercice de la souveraineté y supposera l'inséparable amalgame de ces trois ordres de compétences : religieuses, politiques, juridiques. 
C'est sur ce socle, consacrant dûment la prééminence du religieux, que l'auteur fait reposer toute l'histoire de la Cité antique, et des Cités antiques, au gré des conflits, antinomies, heurs et malheurs de ces différents ordres entre eux, ainsi que de leur relation agonistique avec ces « exclus » de l'origine que sont les non-patriciens à Rome, ou les non-eupatrides à Athènes. C'est sur le statut religieux du culte familial, en premier, que porte la revendication, puisque c'est par lui que passe toute forme de reconnaissance civique. Ce sera vrai jusqu'à ce que la démocratie en déplace provisoirement les enjeux (notamment à Athènes), non sans qu'un Socrate pourtant en meure, au nom même des antiques croyances.

La plèbe à Rome et le dèmos en Grèce tenteront d'opérer d'autres groupements de population, de briser la gens ou le révos, avec une durée ou un succès tout relatifs, selon les lieux, selon les temps. Il faudra en tout cas attendre que d'autres dieux et d'autres croyances permettent aux Stoïciens et aux Chrétiens de parler de liberté intérieure. D'autres formes, sociales et étatiques seront alors possibles, par-delà les archaïques rechutes de la superstition romaine jusqu'à la fin de l'époque impériale.

La notion d'un Dieu transcendant semble la plus originale nouveauté conditionnant les formes d'une ère nouvelle. En résulteront aussi, mais Fustel ne le dit pas, la Querelle des Investitures et l'Inquisition...

Quoi qu'il en soit, la démarche ici n'a rien de déterministe ni de systématique. Pour le lecteur contemporain, Taine et Spengler y sont renvoyés dos à dos. Point de philosophie de l'histoire, donc, pour celui qui nous dit et nous répète que, contrairement à ce que pense Montesquieu, les croyances durables ne peuvent être des impostures, toutes relatives soient-elles.

Il nous rappelle aussi que si l'on peut être tenté d'expliquer les particularismes par le morcellement géographique de la Grèce, des villes aussi irréductiblement opposées que Thèbes et Platées, Argos et Sparte, Sybaris et Crotone n'avaient entre elles aucune chaîne de montagnes (p. 238). Leurs différences municipales résident donc dans les codes différents selon lesquels elles se souviennent différemment de leur fondation et de leurs dieux ou héros tutélaires.

Enfin, la dernière réflexion que je me permettrai sera, encore, d'ordre méthodologique. J'ai rappelé l'importance du retour aux textes pour Fustel. Certes, et l'historien s'y reconnaît. Quant au philologue, il accepte sans peine que les références aux œuvres littéraires d'Hérodote, Thucydide, Démosthène, Cicéron, Tacite ou Juvénal y soient incidemment traitées plutôt en documents historiques qu'en monuments esthétiques. Une chose cependant me frappe : c'est que malgré tant de précision dans la lecture et d'acribie dans la chronologie, les abondantes notes en bas de page nous livrent les unes à côté des autres et comme indifféremment des témoignages d'époques très diverses. Gaius, Ulpien, les «Institutes » y côtoient les deux Pline, Aulu-Gelle et Macrobe, de même que Philocore et Aristophane voisinent couramment avec Pausanias ou Plutarque. Or, lorsque l'auteur s'en justifie, c'est pour nous dire, 
quant à l'archaïsme, que les vieilles formules, religieuses ou juridiques, sont d'une étonnante longévité, frôlant parfois la fossilisation. Je le crois volontiers. D'autant que j'ai moi-même souvent éprouvé dans mes propres recherches d'histoire des religions combien certains textes tardifs pouvaient charrier d'archaïsme parfois inconscient : Ovide et Virgile nous ont gardé à propos des cultes de Cybèle ou de Dionysos des notations infiniment précieuses, que l'on chercherait vainement en amont de leurs ouvres ${ }^{1}$. Et qu'en serait-il de l'orphisme si l'on ne prenait en compte la fameuse et tardive anthropogonie relatée par Diodore et Plutarque, dont le mythème de «conte barbare » ne peut en aucun cas avoir été le fruit d'une invention tardive ${ }^{2}$ ?

Bien sûr, il faut, en cette pratique, raison garder, cultiver le sens critique et comme le rappelait notre maître Labarbe, après Épicharme et Mérimée, « rester sobre et n'oublier point de se défier ».

Numa-Denis Fustel de Coulanges en eût sans aucun doute convenu, lui aussi.

\section{Remarques :} Hachette.

- J'ai relu le texte de la Cité antique dans sa réédition de 1969, Paris,

- Une présentation originale et inattendue en est faite dans le célèbre essai de Jules de Gaultier, Le Bovarysme (Paris, Mercure de France, 1926, p. 143155). Ce "pouvoir départi à l'homme de se concevoir autre qu'il n'est », appliqué par l'auteur à toute l'œuvre de Flaubert, trouve également des applications dans les domaines les plus divers de la culture et de l'histoire, et notamment à ces rites désormais incompréhensibles mais indéfectiblement maintenus dans une "cité antique " aveuglément fidèle à ses anciennes croyances.

Université de Liège

Pierre SOMVILLE

Place du 20-Août, 32

B - 4000 LIÈGE

1 Voir mes Études grecques, Bruxelles-Liège, P. Mardaga, 1990. À propos du symbolisme de l'abeille à la fin du livre IV de Géorgiques, associé aux cultes de la Grande-Mère : "L'Abeille et le taureau » p. 13 et au sujet du rôle analogique tenu par le dauphin dans le culte bacchique clairement perçu par Ovide dans le passage où il nous relate la métamorphose des marins impies : «L'image du dauphin », p. 45-46.

2 Il s'agit des allusions au dépècement, à la cuisson et à la manducation des chairs de l'enfant Dionysos par les Titans : DIOD., III, 62, 7-8; PLUT., De esu camium, I, 996c. 